\title{
My Knowledge Management (MyKM) Guidelines and Video Tutorial: Special Project in Library
}

\author{
Mazwani Ayu binti Mazlan \\ Faculty of Information Management, Universiti Teknologi MARA \\ UiTM Puncak Perdana Campus, Selangor, Malaysia
}

Tel: 603-7962 2063 E-mail: mazwani419@uitm.edu.my

Hamidah binti Mohd Hamdi

Faculty of Information Management, Universiti Teknologi MARA

UiTM Puncak Perdana Campus, Selangor, Malaysia

Tel: 603-7962 2000 E-mail: hamidahhamdi13@gmail.com

Received: June 2, 2021 Accepted: July 10, $2021 \quad$ Published: August 4, 2021

doi:10.5296/ijssr.v9i2.18712 URL: https://doi.org/10.5296/ijssr.v9i2.18712

\begin{abstract}
The goal of this article is to highlight a special project completed by a final semester Bachelor of Information Science (Hons.) Resource Centre Management student at UiTM Shah Alam's Perpustakaan Tun Abdul Razak (PTAR). In addition to the internship activity in the company or organisation, students must complete a special project as an extra task that will contribute to the library. The focus of this article is on a particular project involving a tutorial on how to use the library UiTM's MyKnowledge Management (MyKM) website. The development method was used, with stages such as analysing the learning objectives, creating a storey board, validating the story board, revising the story board, and creating video tutorial media. The article will be discussing about the process of creating the video tutorial and user guidelines.
\end{abstract}

Keywords: PTAR, my knowledge management UiTM, MyKM UiTM, video tutorials, guidelines 


\section{Macrothink

\section{Introduction}

Knowledge management has now become a mainstream priority for businesses of all sizes. According to Bhojaraju, G. (2019), Securing and effectively disseminating a company's most valuable Knowledge (asset) across the enterprise is a business-critical issue for many helps desk, customer support, and IT departments. Abdul Kalam (2004), have earlier stated that the systematic process of finding, selecting, organising, distilling, and presenting information improves an employee's comprehension in a specific area of interest in knowledge management, that will assist the organisation in gaining insight and understanding from its own experiences. Specific knowledge management tasks aid in the acquisition, storage, and utilisation of knowledge for problem solving, dynamic learning, strategic planning, and decision making. It also reduces the deterioration of intellectual assets, improves firm intelligence, and increases flexibility. This is later enhanced by the Gartner Group (2005), that define knowledge management as a discipline that encourages an integrated approach to identifying and managing all of an organization's information assets, that include database records, rules, and processes, as well as individuals' unarticulated skill and experience, are examples of information assets and this will go through process of developing, technical, and organisational infrastructure; all aspects of knowledge management are being done for the purpose of systematic storage and easy retrieval. Intezari, A. et al. (2017) have mentioned that knowledge management encompasses more than just knowledge technology. To achieve strategic business objectives, knowledge management must be an enabler. Attempts to impose new technical infrastructures that are either unsuited for their work settings or where employees are unwilling to share expertise have resulted in a lot of organisational garbage. As a result, the knowledge management effort emerges as a solution to such difficulties, bringing together people, processes, and technology to assist corporations in achieving their goals and vision.

The situation of emergence technology and fast changing trends nowadays had made most of the organizations to reduce cycle times, operate with minimal fixed assets and overhead (people, inventory, and facilities), shorten product development time, improve customer service, empower employees, innovate and deliver high quality products, increase flexibility and adoption, capture information, create knowledge, share, and learn in order to serve customers well and remain relevant in business. None of this is possible unless all employees and teams, at work and in the marketplace, maintain a constant focus on the creation, updating, availability, quality, and use of knowledge. 


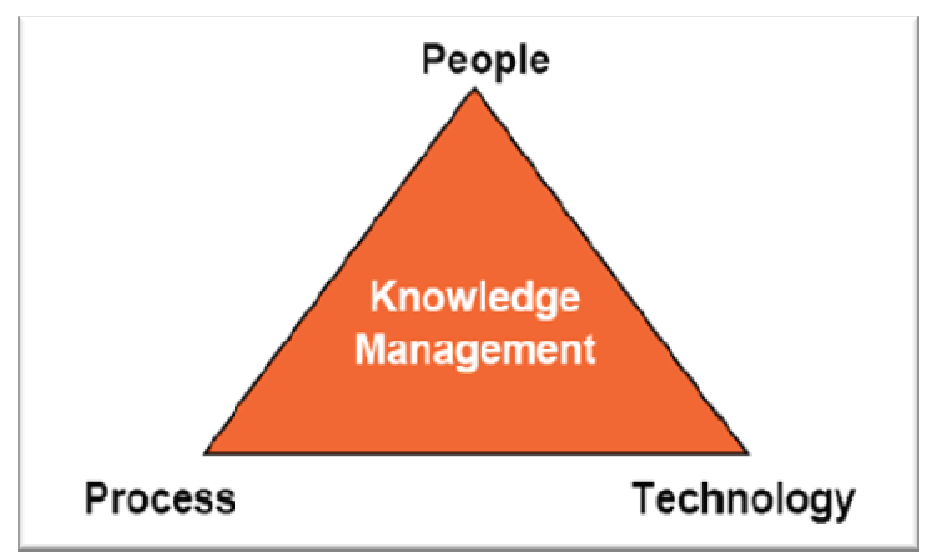

Figure 1. Component of knowledge management

Based on the actual experiences of the world's leading KM case studies, the components of $\mathrm{KM}$ can be broadly classified into three classes: people, processes, and technology (as seen in Figure 1). While all three are necessary for developing a learning organisation and achieving business results from KM, the majority of organisations worldwide that have implemented $\mathrm{KM}$ have found it relatively easier to implement technology and processes. This is the focus of UiTM Library in creating the My knowledge Management (MyKM) where the objectives are to serve people and adapting to as much technology as adherence to the process all together.

\section{My Knowledge Management (MyKM)}

UiTM Library has created the My knowledge Management (MyKM) system to collect, manage, and disseminate knowledge information based on reference materials produced by UiTM authors and researchers as well as the rest of scholar's contributions all around the world. The goal of MyKM is to collect and manage knowledge through the provision of reference materials in various fields that are produced and published either within or outside the university, to promote the element of knowledge transfer and knowledge sharing to improve intellectualism among the university community, and to empower MyKM as a leading reference and user in gaining knowledge transfer and knowledge sharing to improve intellectualism among the university community and empowering $\mathrm{MyKM}$ as a leading reference and reader in gaining knowledge based on research expertise and best practices. This is done to support the library's objectives of providing the best possible services to library users, as well as to contribute to raising awareness of the importance of celebrating knowledge in all forms.

\section{My Knowledge Management (MyKM) Tutorial Video}

Technology alone cannot promote the expected returns because returns are realised by end-users through the use of technology. According to Mmatloa (2020), organisations have realised that deploying a solution is the easy part of supporting KM. This have influenced the establishment of the project in order to further provide a medium for the users to be able to 


\section{I Macrothink}

International Journal of Social Science Research

ISSN 2327-5510

2021, Vol. 9, No. 2

connect with MyKM more effective. When it comes to teaching a procedure or offering step-by-step directions, tutorial videos are the go-to educational tool. Tutorial videos are typically 2-10 minutes long and use a variety of instructional strategies. The greatest instructional videos, sometimes known as "how-to" videos, are well-planned and polished. Video tutorials have been proved to be an effective medium for learning, for example, by providing user-guided experience (Duffy, 2008) and encouraging learners to explore and learn at their own pace (Mullampy, 2010; Duncan, 2013). Video tutorials are useful in offering an introduction to a technology and displaying how a piece of software may be built within an IDE (Integrated Development Environment), according to a study by MacLeod et al. (2015) on programming videos on YouTube. Ponzanelli et al. conducted a developer poll a year later (2016), and found that many developers use video tutorials on a regular basis. The proposals of creating a tutorial video and guideline for the user were accepted by the library after a pitching session on justifying the needs to embrace the user's feedback and informational needs. With these, it is hoped that students or library users will be able to obtain a wealth of information, and this website will be more tailored to the individual who is logged in. The notable part of MyKM is that students can check on their own how many books they have borrowed, what books they still have borrowed, if they have a fine or not, and so on. Every UiTM student should be conscious of and exposed to this website, as it is a novel system with numerous advantages.

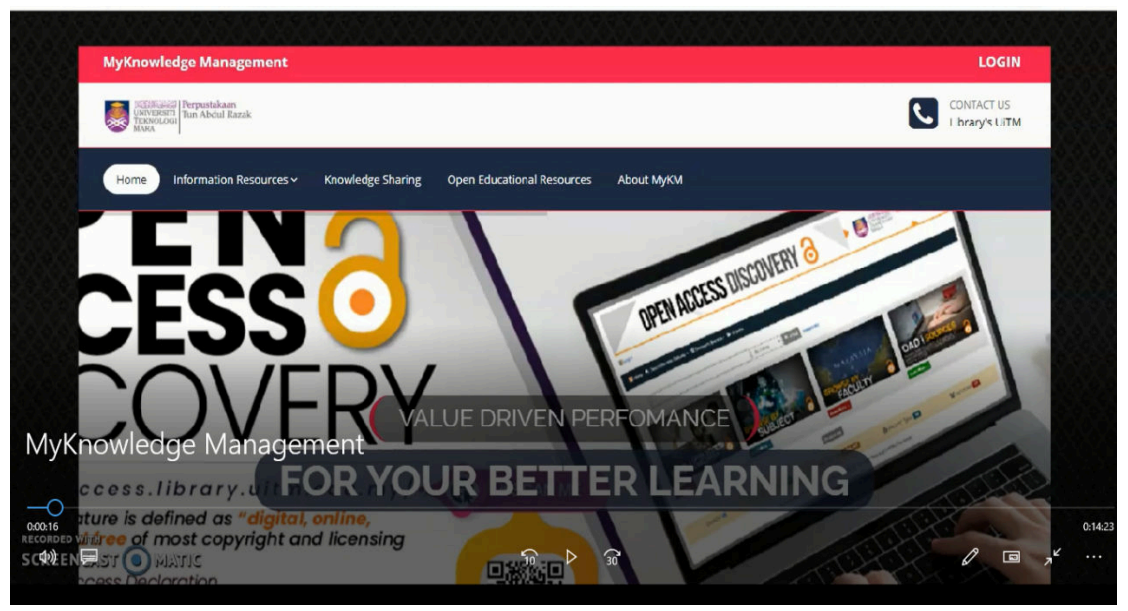

Figure 2. Snapshot of video tutorial my knowledge management (MyKM) opening

The narrator in this video tutorial, which lasts 14:39 minutes, goes over each component of the website, including the home page, information resources, knowledge sharing, open educational resources, and MyKM. There are online databases, E-books, local content hubs, exam papers, khazanah melayu (Malay Heritage) repositories, open access discovery, and institutional repositories amongst some of the information resources. According to Bao, L. (2018), system developers not only require not only conceptual knowledge, for example knowledge about the concepts and APIs involved in the task, but also procedural knowledge, 


\section{Ml Macrothink}

that is all actions and manipulations that apply conceptual knowledge in the task, to complete a programme task. Software artefacts, such as task-created code, keep track of factual information for software development. Although the project does not require the assessing and creating of guidelines for the developer that are more technical, guideline for the user is crucial and important. These strongly suggest that having a tutorial guide will help in completing the task in a programme; as in this case the MyKM.

\subsection{Methodology}

Research indicates that the active generation and elaboration of material explanations is more useful than the passive spending of time reading or listening to teachers, lecturers or tutor ((Graesser, et al., 2005). In developing MyKM guidelines, a strategic plan based on a successful tutorial development are taking place.

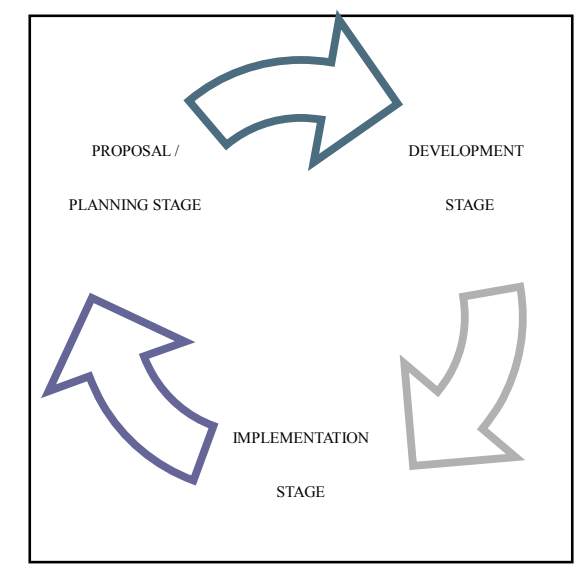

Figure 3. Stages of tutorial development

\subsubsection{Proposal Stage}

During the proposal stage, there are a few vital elements included that is the objectives, the targeted user and layout plan. Objectives in education are brief statements that describe what is the outcomes expected by the user; for MyKM it is to further enhance their comprehension and skills in administering and navigating the sites. During a monthly department meeting, the proposal is presented to the ICT department supervisor with the presence of the organization's heads. After a pitching session the library accepted the proposals for creating a video tutorial and user guidelines justifying the user needs in terms of feedback and information.

\subsubsection{Development Stage}

A layout plan was used to develop the video tutorial (see Figure 4). Content determination is carried out with copywriting as it is aimed at persuading a reader to undertake a specific action, specifically for this project as it will ensure that the script establishing a connectivity 
towards the user. Users can understand more if they present the tutorial in a layman manner and warm atmosphere with appropriate supporting media (Hampel \& Hauck, 2004). The recording is done later in the development stage, when the content has been determined.

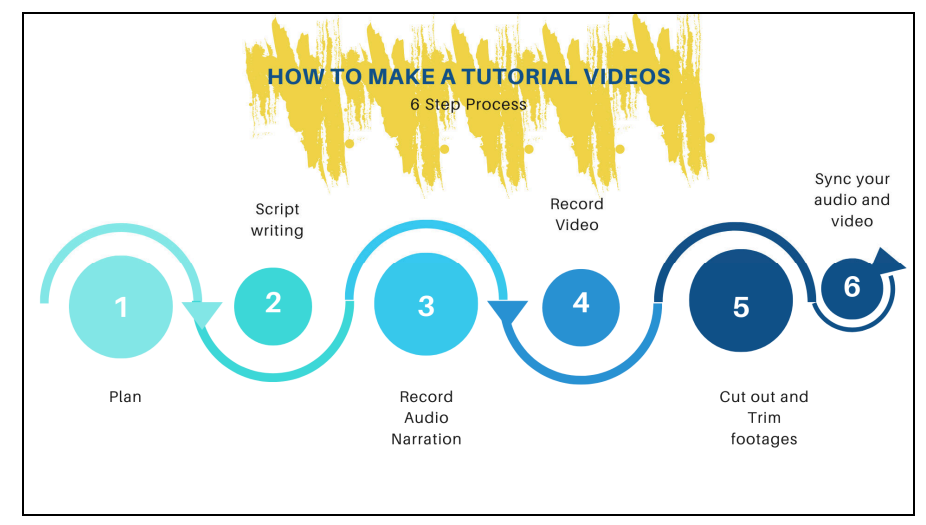

Figure 4. 6 Step Process of how to make tutorial videos

Source: Techsmith.com.

\subsubsection{Implementation Stage}

Choose a share destination and a location for this video. For this project it is embedded within the MyKM sites that are administered by the ICT team. It is suggested that as a final step to share the video with a few people to solicit feedback (Frick \& Eyler-Werve, 2014). This is an excellent technique for ensuring that your message is clear and that your video is accomplishing its goals.

\section{The MyKM Guidelines in PDF}

According to Cambridge.org (2019), a guideline is intended to streamline specific operations in accordance with a predetermined routine or best practise. Any business (public or private) can establish and apply guidelines to make the behaviour of its employees or divisions more predictable and, presumably, of greater quality. A guideline resembles a rule. Therefore, although there is a video tutorial designed for MyKM, it is also important to have in a written form as the users of the MyKM may choose between this two, according to their preferences. Susan Aitchinson (2019) have created a survey on preferences of video tutorial and written guidelines, there are some have mentioned that written tutorials are preferred since it allow them to learn at their own pace rather than at the pace of the video. Emily Cotlier (2019) have discussed the advantages of using written guidelines rather than video tutorials. First, writing instructions is easier to generate and update than making a high-quality video. Second, it's easier to translate written instructions into a different language than it is to translate spoken instructions. Lastly, users can quickly locate the piece of instructions that they require. The guideline created are basically a screenshot of every webpage and compiled accordingly in a PDF form. Below is the table of process taken to develop the guidelines (Table 1). 


\section{Macrothink \\ International Journal of Social Science Research \\ ISSN 2327-5510 \\ 2021, Vol. 9, No. 2}

Table 1. Process of establishing guideline

TASK ACTIVITY

Task 1 Gathering all the necessary information and materials, study through the MyKM website

Task 2 Draft a proposal and mock up to establish a guideline

Task 3 Develop the guideline

Task 4 Embed guideline to the website

The guidelines are established using the Web Content Accessibility Guidelines (WCAG) mentioned by Comperverde-Molina, et al. (2020), are guidelines for web accessibility developed by the Web Accessibility Initiative and the World Wide Web Consortium. These recommendations state, in general, that websites must be secure.

- Perceivable: Visitors are aware of the content on your site.

- Operable: The functionality of your website should be possible in different ways.

- Understandable: All content and alerts can be easily understood.

- Robust: Your website is usable across different assistive technologies, devices, and browsers.

The goal here is to create a website that anyone can use, including people with disabilities or other limitations. The language and structure of the sentence used in a MyKM guideline is also important. Although experienced employees can often be relied on to bend rules to their advantage, there may be some guidelines from which no deviation is permissible, particularly in the areas of security and branding. A phrase that encompasses such a broad range of meaning is therefore necessary. Brown, G. (2018) mentioned that using the definitions developed by Scott Bradner at the Internet Engineering Task Force (IEFT) is a good way to accomplish this. The IEFT has produced a number of terms that indicate the strictness by which particular rules must be respected. By using these, a high degree of confidence about how guidelines are interpreted may be established.

These terms are:

- Must: This word indicates an absolute requirement that cannot be ignored.

- Must Not: This phrase indicates an absolute prohibition that cannot be ignored.

- Should: This word means that valid reasons may exist to ignore an item, but the full implications must be understood and carefully weighed before choosing a different course. 
- Should Not: This phrase means that there may exist valid reasons when a particular behaviour is acceptable or even useful, but the full implications should be understood and the case carefully weighed before implementation.

- May: This word means that an item is truly optional.

Merging these wording guidelines with the document structure already indicated, it is possible to imagine how a website 'manual' might look. for example, in developing MyKM, it is divided into five sections (as in Figure 5)

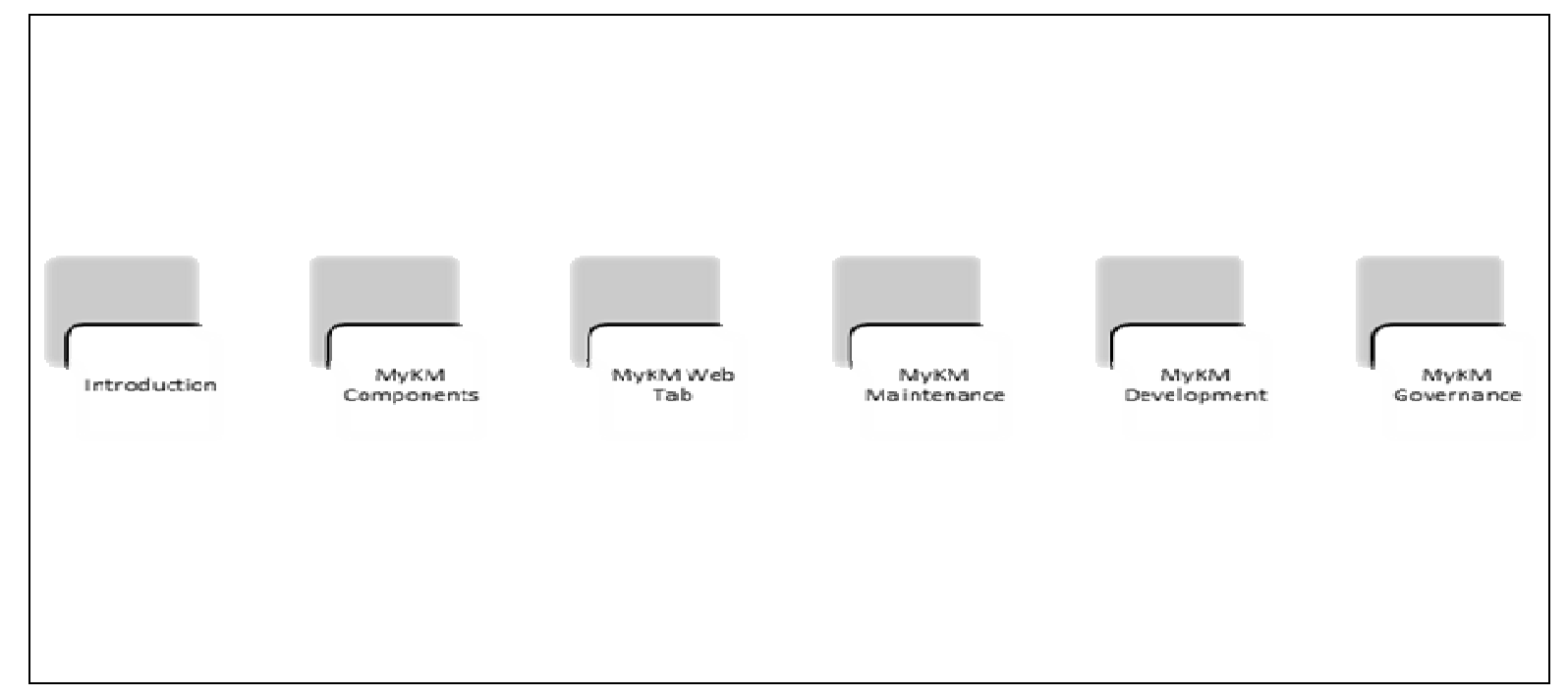

Figure 5. Sections in MyKM Guideline

The sections of MyKM Guideline are developed as a guidance of a mainframe of the actual guideline contents. It is as per details provided below:

\subsection{Introduction}

The introduction's purpose is to explain the overall goal of the guideline. It could also include suggestions for document sections that are appropriate for different audiences. Librarians, for example, should be familiar with library governance rules, whereas developers and designers will benefit most from development guidelines (not in the scope of the project)

\subsection{MyKM Components}

This section provides the content arrangement of MyKM. This is to ensure that the guidelines are synchronize with the webpage interface.

- Homepage

- Information Resources

- Online Database 


\section{Macrothink

- Local Content Hub

- Business Fundamentals

- Download

- Journal

- EQPS

- Repositori Khazanah Melayu

- Open Access Discovery

- Institution Repository

- Knowledge Sharing

- MyCourseware

\subsection{MyKM Web Tab}

MyKM Tab is the features of the website that enable to right-click the content desired without having to leave the current page. These are the pages that enable a right-click function;

- Online database

- Download

- Instituitional Respository

- $\quad$ Repositori Khazanah Melayu

\subsection{MyKM Maintenance}

This section outlines the rules that must be followed for maintenance activities. As an example:

"To ensure that the intranet is available and functioning properly, it must be checked for Quality Assurance issues. This task should be completed weekly, but at the very least monthly “

\subsection{MyKM Governance}

This section outlines the most stringent rules for running a site. It could also include an overview of the factors that influence and necessitate such structures, such as the law, company policies, technology, and so on. For example:

"The following legislation must be respected when making a change of any kind to the website: Data Protection Act, Criminal Damage Act, Electronic Commerce Act”. 


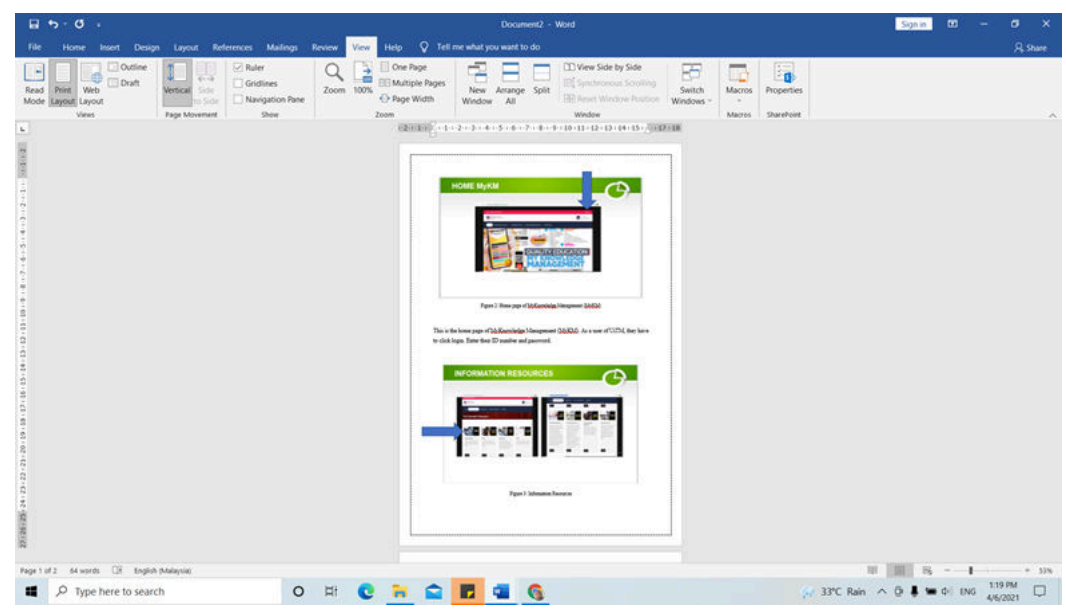

Figure 6. A screenshot of creating the PDF guideline in word doc. for MyKM

After that, the draft of guidelines is made in a word document (as seen in Figure 6) before converting it into a PDF (as seen in Figure 7). It is a straight forward procedure that does not need any additional software for the process of emerging word document to PDF.

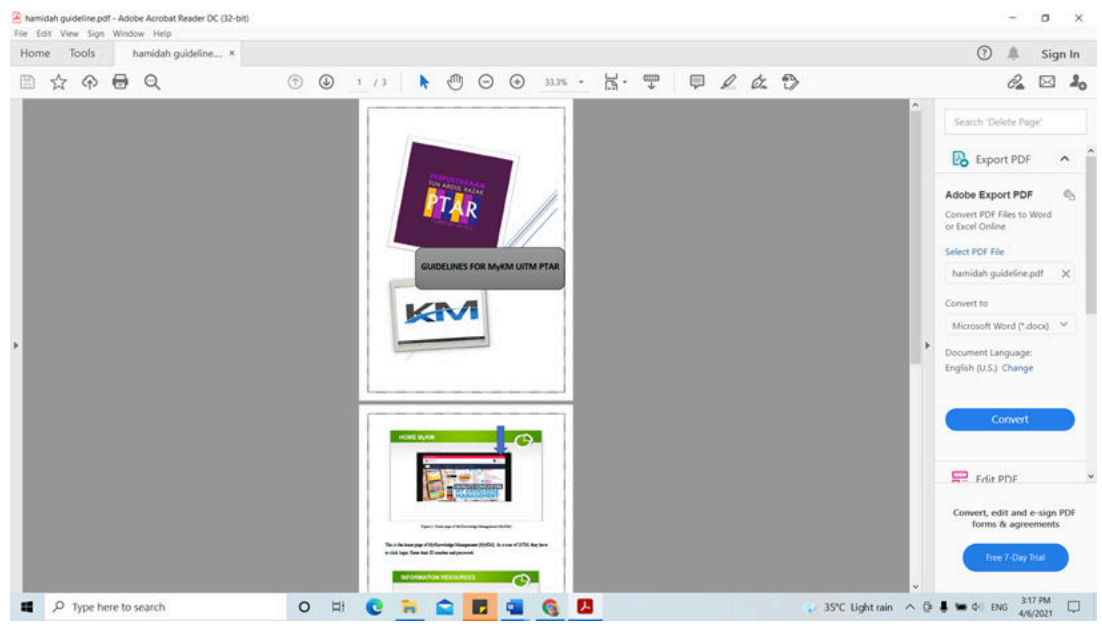

Figure 7. A screenshot of creating the PDF guideline for MyKM

\section{Conclusion}

Creating a guideline and video tutorial for MyKM provides more insight into how the website works and could benefit users who are aware of the website's existence greatly. The library has raised a lot of awareness, and the response has been positive. The experience gain from this is there are many ways to be adapted in creating the guideline and tutorial videos. However, with further research and professional analysing helps in choosing the right process within the capabilities of an inexperience website admin. It can be suggested that the MyKM admin master shall take a serious look into this and come up with a more interactive and 
creative looking tutorial that usually takes a lot of effort and more time given. Library can also play an important part of scrutinizing more details that are needed by conducting an UX survey in order to understand and take actions on user feedback for MyKM usage. Finally, My Knowledge Management (MyKM) is a new system website that provides a wealth of information to users, particularly students. As students, they should seize the opportunity to use this system because it provides numerous benefits and is extremely beneficial. This research is one method of promoting the My Knowledge Management (MyKM) website to the readers. They can learn and experiment with this website.

\section{Acknowledgments}

It is with deepest gratitude express to the Universiti Teknologi MARA library, Perpustakaan Tun Abdul Razak (Tun Abdul Razak Library) for the opportunity given for internship at the organization. Special thanks to Faculty of Information Management for support and encouragement during the duration of establishing this special project.

\section{References}

Abdul Kalam, A. P. J. (2004). Digital Library and its multidimensions. President of India's speech at the "Inauguration of International Conference on Digital Libraries (ICDL), New Delhi: Feb 24, 2004.

Bao, L., Xing, Z., Xia, X., \& Lo, D. (2018). Vt-revolution: Interactive programming video tutorial authoring and watching system. IEEE Transactions on Software Engineering, 45(8), 823-838. https://doi.org/10.1109/TSE.2018.2802916

Brown, G. (2018). Internet Engineering Task Force (IETF) J. Gould Request for Comments: 8334 VeriSign, Inc. Category: Standards Track W. Tan.

Cambridge English Dictionary. Retrieved from http://www.dictionary.cambridge.org

Campoverde-Molina, M., Luján-Mora, S., \& García, L. V. (2020). Empirical studies on web accessibility of educational websites: A systematic literature review. IEEE Access, 8, 91676-91700. https://doi.org/10.1109/ACCESS.2020.2994288

Duffy, P. (2008). Engaging the youtube google-eyed generation: Strategies for using web 2.0 in teaching and learning. The Electronic Journal of e-Learning, 6(2), 119-130.

Duncan, L., Yarwood-Ross, \& Haigh, C. (2013). Youtube as a source of clinical skills education. Nurse Education Today, 33(12), 1576-1580. https://doi.org/10.1016/j.nedt.2012.12.013

Elmetwaly, A. A. (2021). Instructional Guidelines for Patients with Pott's Fracture to Improve Physical Activity and Functional Ability. Mansoura Nursing Journal, 8. https://doi.org/10.21608/mnj.2021.58394.1003

Emily, C. (2019). What's best for instructions-words or videos? Retrieved from https://write.co.nz/

Frick, T., \& Eyler-Werve, K. (2014). Return on engagement: Content strategy and web design techniques for digital marketing. Routledge. https://doi.org/10.4324/9780203751817

GartnerGroup. (2005). Retrieved from http://www.gartner.com

Graesser, A. C., McNamara, D. S., \& VanLehn, K. (2005). Scaffolding deep comprehension 
strategies through Point \& Query, AutoTutor, and iSTART. Educational Psychologist, 40(4), 225-234. https://doi.org/10.1207/s15326985ep4004_4

Gunjal, B. (2019). Knowledge management: Why do we need it for corporates. Malaysian Journal of Library \& Information Science (ISSN: 1394-6234). https://doi.org/10.31229/osf.io/fy984

Hampel, R., \& Hauck, M. (2004). Towards an effective use of audio conferencing in distance language courses. Language Learning \& Technology, 8(1), 66-82.

Intezari, A., Taskin, N., \& Pauleen, D. J. (2017). Looking beyond knowledge sharing: An integrative approach to knowledge management culture. Journal of Knowledge Management, 21(2). https://doi.org/10.1108/JKM-06-2016-0216

Library Universiti Teknologi MARA. (n.d.). UiTM Library. Retrieved from https://library.uitm.edu.my/en/

MacLeod, L., Storey, M.-A., \& Bergen, A. (2015). Code, camera, action: How software developers document and share program knowledge using Youtube (pp. 104-114). In Proceedings of 23rd IEEE International Conference on Program Comprehension (ICPC). IEEE Press, 2015. https://doi.org/10.1109/ICPC.2015.19

Mmatloa, P. L. (2020). Guidelines to improve the use of knowledge management systems as intended: the perspective of end-users. Doctoral dissertation, North-West University (South Africa).

Mullamphy, D., Higgins, P., Belward, S., \& Ward, L. (2010). To screencast or not to screencast. Anziam Journal, 51, C446-C460. https://doi.org/10.21914/anziamj.v51i0.2657

MyKnowledge Management. (2020). MyKnowledge Management.

Ponzanelli, L., Bavota, G., Mocci, A., Di Penta, M., Oliveto, R., Hasan, M., ...Lanza, M. (2016). Too long; didn't watch!: Extracting relevant fragments from software development video tutorials (pp. 261-72). In Proceedings of the 38th International Conference on Software Engineering (ICSE), ACM, 2016. https://doi.org/10.1145/2884781.2884824

Susan, A. (2019). Do you prefer video or written coding tutorials? The Dev:to Forum

\section{Copyrights}

Copyright for this article is retained by the author(s), with first publication rights granted to the journal.

This is an open-access article distributed under the terms and conditions of the Creative Commons Attribution license (http://creativecommons.org/licenses/by/4.0/). 